\title{
Effects of periodontitis on aortic insulin resistance in an obese rat model
}

\author{
Daisuke Ekuni ${ }^{1}$, Takaaki Tomofuji ${ }^{1}$, Koichiro Irie ${ }^{1}$, Kenta Kasuyama ${ }^{1}$, Michihiro Umakoshi ${ }^{1}$, Tetsuji Azuma ${ }^{1}$, \\ Naofumi Tamaki ${ }^{1}$, Toshihiro Sanbe ${ }^{1}$, Yasumasa Endo ${ }^{1}$, Tatsuo Yamamoto ${ }^{2}$, Takashi Nishida ${ }^{3}$ and Manabu Morita ${ }^{1}$
}

The combination of obesity and its associated risk factors, such as insulin resistance and inflammation, results in the development of atherosclerosis. However, the effects of periodontitis on atherosclerosis in an obese body remain unclear. The aim of the study was to investigate the effects of ligature-induced periodontitis in Zucker fatty rats on initiation of atherosclerosis by evaluating aortic insulin resistance. Zucker fatty rats $(n=24)$ were divided into two groups. In the periodontitis group, periodontitis was ligature-induced for 4 weeks, whereas the control group was left unligated. After the 4-week experimental period, descending aorta was used for measuring the levels of lipid deposits, immunohistochemical analysis, and evaluation of gene expression. Levels of serum C-reactive protein (CRP), tumor necrosis factor$\alpha(T N F-\alpha)$, and insulin were also measured. Rats in the periodontitis group had significantly enhanced lipid deposits in the aorta, but not in the control group. Expression of suppressor of cytokine signaling 3, vascular cell adhesion molecule 1, reactive oxygen species, nitrotyrosine, and endothelin-1 in the periodontitis group was more intense than that in the control group. Significantly decreased levels of phosphatidylinositol 3-kinase (Pi3k) catalytic $\beta$-polypeptide (Pi3kcb), Pi3kp85, and insulin receptor substrate 1 and 2 were observed in the periodontitis group. Levels of serum CRP and TNF- $\alpha$ were significantly increased in the periodontitis group. Under insulin-stimulated conditions, aorta in the periodontitis group altered the Akt phosphorylation. Periodontitis in obesity induced the initial stage of atherosclerosis and disturbed aortic insulin signaling.

Laboratory Investigation (2010) 90, 348-359; doi:10.1038/labinvest.2009.141; published online 11 January 2010

KEYWORDS: animal models; atherosclerosis; insulin resistance; obesity; periodontal disease

Obesity has rapidly become a worldwide disease. The pathophysiological consequences of obesity include insulin resistance, hypertension, and dyslipidemia, which are referred to as the metabolic syndrome. ${ }^{1}$ Aside from these primary conditions associated with obesity, risk factors such as increased markers of inflammation, thrombosis, oxidative stress, and elevated plasma leptin levels are all associated with the pathological conditions related to obesity. ${ }^{2}$ The combination of obesity and its associated risk factors results in the development of cardiovascular diseases such as atherosclerosis. ${ }^{3}$

Insulin resistance at a systemic level is an independent risk factor for the development of atherosclerosis. ${ }^{4-7}$ One of the possible mechanisms linking insulin resistance to atherosclerosis is endothelial dysfunction, ${ }^{8}$ which is supported by a number of studies. ${ }^{9-12}$ Endothelial dysfunction refers to an imbalance in the release of vasodilating factors such as nitric oxide (NO) and vasoconstricting factors such as endothelin-1 (Et-1). ${ }^{9}$ Insulin has both a vasodilatory effect through upregulation of NO production via stimulation of phosphatidylinositol 3-kinase (Pi3k)/Akt pathway and a vasoconstrictory effect through Et-1 production via MAP kinase pathway. ${ }^{9}$

Periodontitis is one of the most widespread inflammatory diseases, characterized by gingival bleeding, periodontal pocket formation, destruction of connective tissue attachment, and alveolar bone resorption. ${ }^{13,14}$ Many studies have demonstrated that atherosclerosis and periodontitis are associated and possibly causally linked. ${ }^{15-19}$ Therefore, it is possible that periodontitis augments the effects of obesity on

\footnotetext{
${ }^{1}$ Department of Preventive Dentistry, Okayama University Graduate School of Medicine, Dentistry and Pharmaceutical Sciences, Okayama, Japan; ${ }^{2}$ Division of Sociological Approach in Dentistry, Department of Dental Sociology, Kanagawa Dental College, Yokosuka, Japan and ${ }^{3}$ Department of Biochemistry and Molecular Dentistry, Okayama University Graduate School of Medicine, Dentistry and Pharmaceutical Sciences, Okayama, Japan

Correspondence: Dr T Tomofuji, PhD, Department of Preventive Dentistry, Okayama University Graduate School of Medicine, Dentistry and Pharmaceutical Sciences, 2-5-1 Shikata-cho, Kita-ku, Okayama 700-8525, Japan.

E-mail: tomofu@md.okayama-u.ac.jp

Received 9 July 2009; revised 12 November 2009; accepted 23 November 2009
} 
atherosclerosis. However, the mechanistic relationship between atherosclerosis and periodontitis in obesity remains unclear.

We hypothesized that periodontitis may have additive effects on the pathogenesis of atherosclerosis in obesity by disturbing aortic insulin resistance. The Zucker fatty rat was chosen as it is a well-established model of prediabetes and characterized by hyperphagia, hyperlipidemia, obesity, insulin resistance, hyperinsulinemia, and impaired glucose tolerance that result from a mutation in the leptin receptor gene. ${ }^{20}$ Hence, the aim of the study was to investigate the effects of ligature-induced periodontitis in Zucker fatty rats on initiation of atherosclerosis by evaluating aortic insulin resistance. The differences in aortic gene expression pattern related to metabolic syndrome between obesity rats with and without periodontitis were evaluated using microarray technology. Furthermore, the vasodilating factors such as levels of Pi3k and Akt, the vasoconstricting factors such as Et-1, and the adhesion-related factors such as vascular endothelial growth factor (Vegf) and vascular cell adhesion molecule 1 (Vcam1) were investigated for the endothelial dysfunction. ${ }^{21}$

\section{MATERIALS AND METHODS Experimental Design}

All experimental procedures were performed in accordance with regulations of the Animal Research Control Committee of Okayama University Dental School. Twenty-four male Zucker fatty rats (5 weeks old) were divided into two groups of 12 rats each: the control group and the periodontitis group. Two groups had free access to powdered food (MF, Oriental Yeast, Osaka, Japan) and fresh drinking water daily. Periodontitis was ligature-induced for 4 weeks in the periodontitis group, whereas the control group was left unligated. A cotton ligature was placed in a subgingival position of the mandibular first molars. ${ }^{22}$ For evaluating the phosphorylation of Akt (p-Akt) at Ser473 occurring at the insulin signal-transduction pathway, 12 Zucker fatty rats; the control ( $n=6$ out of 12) and periodontitis ( $n=6$ out of 12) groups, further received insulin administration. ${ }^{23}$ In these rats, $0.1 \mathrm{ml}$ of saline $(0.9 \% \mathrm{NaCl})$ with insulin $(50 \mathrm{IU} / \mathrm{kg})$ (Sigma-Aldrich, St Louis, MO, USA) was injected through the cava vein at the end of experimental period. Then, the descending aorta was removed $5 \mathrm{~min}$ after injection and kept at $-80^{\circ} \mathrm{C}$ until analysis.

\section{Analysis of Periodontal Tissues}

The animals were killed after the experimental period under general anesthesia. Samples of the left mandibular molar regions were resected from each rat and immediately fixed in $4 \%$ paraformaldehyde in $0.1 \mathrm{~mol} / \mathrm{l}$ phosphate buffer ( $\mathrm{pH} 7.4$ ). Mandibular samples were further decalcified with $10 \%$ tetrasodium-EDTA aqueous solution $(\mathrm{pH}$ 7.4) for 8 weeks at $4^{\circ} \mathrm{C}$. Formalin-fixed tissue samples were embedded in paraffin and stained with hematoxylin and eosin. Immuno- histochemical staining of tumor necrosis factor- $\alpha$ (TNF- $\alpha)$ was carried out using the streptavidin-biotin-peroxidase method. ${ }^{22}$ The goat polyclonal antibody against TNF- $\alpha$ (R\&D Systems, Minneapolis, MN, USA) was diluted 1:200 in phosphate-buffered saline. The color was developed with 3-3'-diamino bentizine tetrahydrochloride (DAB), and sections were counterstained with Mayer's hematoxylin.

A single examiner, blind to the treatment assignment, performed the following histometric analyses using a light microscope. The polymorphonuclear leukocytes of the connective tissue subjacent to the junctional epithelium in two standard areas $(0.05 \mathrm{~mm}$ (depth) $\times 0.1 \mathrm{~mm}$ each) were counted under a magnification of $\times 400 .{ }^{24}$ As an indicator of alveolar bone resorption, the distance between the cementoenamel junction and the alveolar bone crest was measured with a microgrid at a magnification of $\times 200 .{ }^{24}$ The number of TNF- $\alpha$-positive fibroblasts and total fibroblasts in five standard areas $(0.1 \mathrm{~mm} \times 0.1 \mathrm{~mm}$ each $)$ adjacent to the cementum within the periodontal ligament were determined, and the ratios of TNF- $\alpha$-positive fibroblasts to total fibroblasts were calculated. ${ }^{22}$

\section{Microarray Expression Analysis}

The descending aorta was harvested, immediately frozen, and kept at $-80^{\circ} \mathrm{C}$ until it was processed for immunohistochemical, microarray, or real-time RT-PCR analyses. Total RNA was isolated from the aorta biopsy samples, which were pooled from four rats in each group, using Trizol reagent (Invitrogen, Carlsbad, CA, USA), according to the manufacturer's instructions. The isolated RNA was quantified by measuring the absorbance at $260 \mathrm{~nm}$ and the purity was determined by the $260 / 280 \mathrm{~nm}$ absorbance ratio. Samples with a ratio of $>1.8$ were used in the procedure described below. ${ }^{19}$

Total RNA samples $(10 \mu \mathrm{g})$ were analyzed using GeneSQUARE, multiple assay DNA microarray metabolic syndrome for rat (Kurabo Industries, Osaka, Japan). Alexa Fluor 555-labeled cDNA was prepared from total RNA from rat tissue through cDNA synthesis, and in vitro transcription was performed using GeneSQUARE cDNA Direct Labeling System (Kurabo Industries), according to the manufacturer's protocol. The microarray slides were first pretreated with a blocking solution consisting of $4 \times$ standard saline citrate (SSC), $0.5 \%$ SDS, and $1 \%$ bovine serum albumin at $42^{\circ} \mathrm{C}$ for $45 \mathrm{~min}$. The labeled cDNA in hybridization buffer (consisting of $2 \times$ SSC, $4 \times$ Denhardt's solution (Sigma-Aldrich), and salmon sperm DNA (Invitrogen, Carlsbad, CA, USA)) were denatured at $95^{\circ} \mathrm{C}$ for $2 \mathrm{~min}$ and cooled to $25^{\circ} \mathrm{C}$. Then, cDNA were applied to each individual array window and hybridized at $65^{\circ} \mathrm{C}$ for $16 \mathrm{~h}$. After hybridization, the solutions of labeled cDNA on the microarray slides were flushed away by a solution containing $2 \times$ SSC and $0.1 \%$ SDS, and then the microarray slide was immediately washed in the following solutions: $2 \times$ SSC and $0.1 \%$ SDS at $25^{\circ} \mathrm{C}$ for $5 \mathrm{~min}, 0.2 \times$ SSC and $0.1 \%$ SDS at $25^{\circ} \mathrm{C}$ for $5 \mathrm{~min}, 0.2 \times$ SSC and $0.1 \%$ 
SDS at $55^{\circ} \mathrm{C}$ for $5 \mathrm{~min}, 0.2 \times \mathrm{SSC}$ at $25^{\circ} \mathrm{C}$ for $1 \mathrm{~min}$, and $0.05 \times \mathrm{SSC}$ at $25^{\circ} \mathrm{C}$ for $2 \mathrm{~min}$. The images for the hybridized array were captured by GenePix4000B microarray scanner (Molecular Devices, Sunnyvale, CA, USA) and quantified using the Genepix Pro 6.0 software (Molecular Devices). The adjusted intensity equals the intensity of each gene minus the background value. The genes with an adjusted intensity of less than twofold the background value were not detected. The normalized intensity to Gapdh gene was calculated by the following formula and compared with other treatment: normalized intensity $=(X-Z) /(Y-Z) \times 10000$, where $X$ is the adjusted intensity of target gene, $Y$ is the adjusted intensity of Gapdh gene, and $Z$ is the median of adjusted intensities of the negative controls.

\section{Real-Time PCR}

Total RNA $(2 \mu \mathrm{g})$ was reverse transcribed by AMV Reverse Transcriptase (TAKARA) at $42^{\circ} \mathrm{C}$ for $30 \mathrm{~min}^{25}$ The cDNA prepared as stated above was diluted 10-fold with yeast RNA $(10 \mu \mathrm{g} / \mathrm{ml})$. Real-time (RT) PCR was performed using TOYOBO SYBR Green PCR Master Mix (TOYOBO, Osaka, Japan) in a LightCycler system (Roche, Basel, Switzerland) for 45 cycles. Reactions were performed in $20 \mu$ l containing $2 \mu \mathrm{l}$ of cDNA, $5 \mu \mathrm{M}$ of each primer, and $1 \times$ SYBR Green master mix. The primer sets and optimized conditions for the PCR of each target (Gapdh, suppressor of cytokine signaling 3 (Socs3), Vegf, Pi3k catalytic $\beta$-polypeptide (Pi3kcb), Vcam1, and Et-1) are listed in Table $1 .{ }^{26-30}$ The absence of the nonspecific PCR products was checked with a melting curve and electrophoresis analysis. The relative copy numbers were computed on the basis of the data obtained with a serial dilution of a representative sample for each target gene.

\section{Histological Evaluation of Aorta Samples}

The remaining descending aorta was stained with oil red $\mathrm{O}$, hematoxylin and eosin, and the methods are described as follows. ${ }^{31}$ Immunohistochemical staining for Socs3, Vcam1, insulin receptor substrate (Irs) 1, Irs2, Pi3kp85, nitrotyrosine, or p-Akt was performed using Histofine Simple Stain MAX PO kits (Nichirei, Tokyo, Japan). ${ }^{31}$ Briefly, aortic tissues were immersed in methanol containing $0.3 \%$ hydrogen peroxide for $30 \mathrm{~min}$ to block endogenous peroxidase activity. The sections were then treated at $4^{\circ} \mathrm{C}$ with an anti- Socs3 (Santa Cruz Biotechnology, CA, USA) ${ }^{32}$ (diluted 1:50), anti-Vcam1 (Santa Cruz Biotechnology) $^{33}$ (diluted 1:100), anti-Irs1 (Signalway Antibody, Pearland, TX, USA) (diluted 1:100), anti-Irs2 (Upstate Biotechnology, Lake Placid, NY, USA) ${ }^{34}$ (diluted 1:100), anti-Pi3kp85 (Santa Cruz Biotechnology) $)^{35}$ (diluted 1:100), anti-nitrotyrosine (Upstate Biotechnology) ${ }^{31}$ (diluted 1:50) or p-Akt (Cell Signal Technology, Beverly, MA, USA) ${ }^{36}$ (diluted 1:50) overnight, followed by treatment with a secondary antibody $\left(\mathrm{Fab}^{\prime}\right)$ with peroxidase complex for $30 \mathrm{~min}$. The color was developed with a solution of $\mathrm{DAB}$ in $50 \mathrm{mmol} / \mathrm{l}$ Tris-HCl buffer ( $\mathrm{pH}$ 7.5) containing $0.001 \%$ hydrogen peroxide and sections were counterstained with Mayer's hematoxylin. Control sections included buffer alone or nonspecific purified rabbit or goat immunoglobulin G. The percentage of aortic lumen that was Socs3-, Vcam1-, Irs1-, Irs2-, Pi3kp85-, nitrotyrosine-, or p-Akt-positive was calculated using computer-assisted image analysis software (WinROOF, Mitani, Fukui, Japan). ${ }^{31}$

\section{Measurement of Reactive Oxygen Species Expression in Aorta}

Reactive oxygen species (ROS) contributes to a reduction in the bioavailability of $\mathrm{NO}^{21} \mathrm{H}_{2} \mathrm{O}_{2}$ may activate redox

Table 1 Primers and experimental conditions for real-time PCR

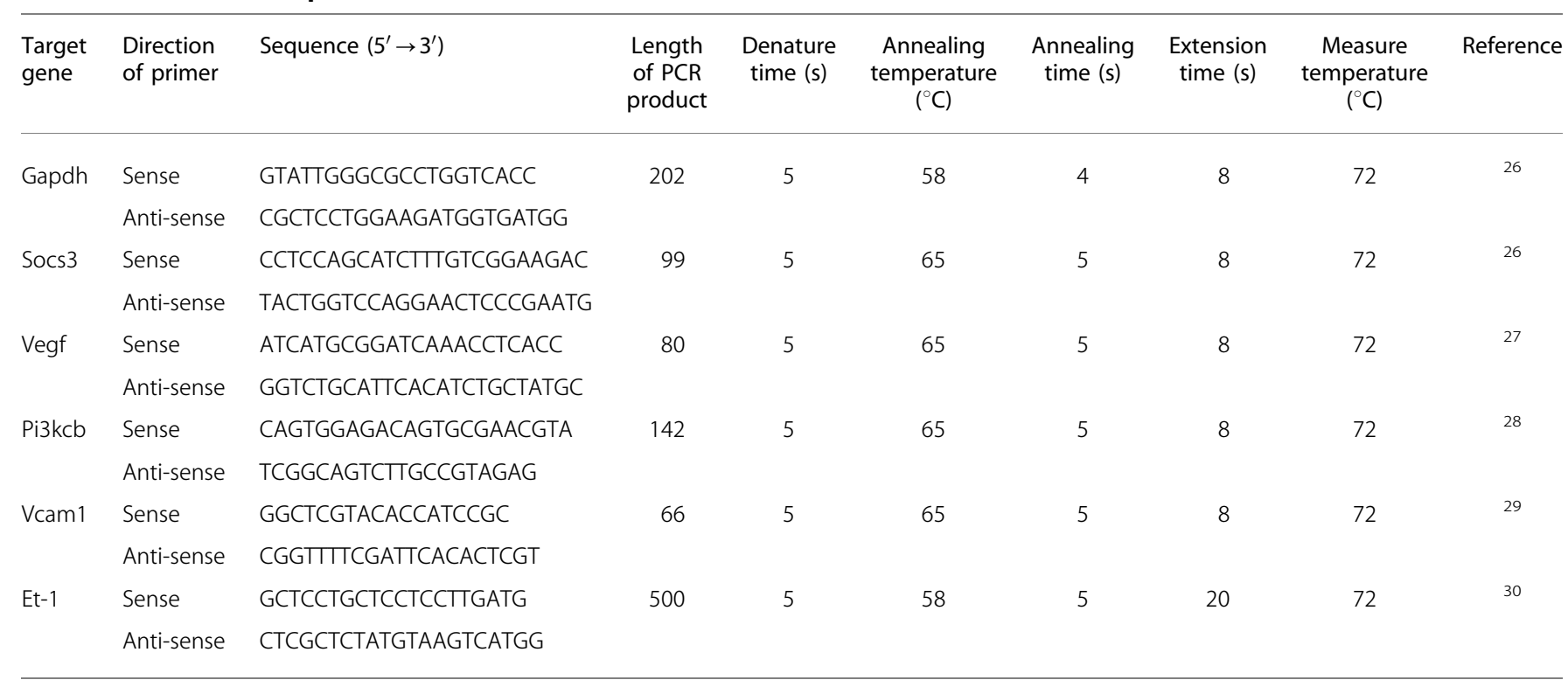


signaling cascades that induce deleterious changes in endothelial cell phenotype. ${ }^{21} \mathrm{DAB}$ staining for the detection of $\mathrm{H}_{2} \mathrm{O}_{2}$ was performed with a modified method. ${ }^{19}$ Briefly, cryostat sections were dried for $5 \mathrm{~min}$ and incubated with Tris- $\mathrm{HCl}(\mathrm{pH} 7.6)$ for $5 \mathrm{~min}$ at $25^{\circ} \mathrm{C}$, followed by a 30 -min incubation in DAB solution (0.1 M Tris- $\mathrm{HCl}, \mathrm{pH} 7.6,0.5 \mathrm{mg} / \mathrm{ml}$ $\mathrm{DAB})$ at $37^{\circ} \mathrm{C}$ then washed with distilled water, the sections were counterstained with Mayer's hematoxylin.

\section{Blood Collection and Measurements of TNF- $\alpha$, C-Reactive Protein, and Insulin Levels}

At the end of the experimental period, blood samples were collected directly from the heart of the animals, which had fasted for $24 \mathrm{~h}$. Blood was allowed to clot at room temperature, and serum was separated by centrifugation at $1500 \times g$ for $15 \mathrm{~min}$. Serum TNF- $\alpha$ concentration was determined with a rat TNF- $\alpha$ enzyme-linked immunosorbent assay (ELISA) kit (Biosource International, Camarillo, CA, USA). ${ }^{37}$ Serum levels of C-reactive protein (CRP) were quantified by a highly sensitive ELISA kit (Life Diagnostics, West Chester, UK) ${ }^{37}$ Serum levels of insulin were quantified by an ultrasensitive ELISA kit (Mercodia, Uppsala, Sweden). ${ }^{38}$ The assays were performed in triplicate.

\section{Statistical Analysis}

Mann-Whitney $U$-test was used for pairwise comparisons between the control and periodontitis groups. All analyses were performed using a software program. $P<0.05$ was considered to be statistically significant.

\section{Sample Size Calculation}

Sample size was calculated by a statistical software (nQuery Advisor, Statistical Solutions, Sangus, MA, USA), based on the results of our previous study. ${ }^{31} \mathrm{~A}$ sample size of six per group was required for detection of a significant difference (80\% power, two-sided 5\% significance level).

\section{RESULTS}

At the end of the experimental period, the mean (s.d.) body weights of the control and periodontitis groups were 342 (42) g and 329 (9) g, respectively. There was no significant difference in the body weight between the control and periodontitis groups.

In the periodontal tissue, the periodontitis group showed destruction of junctional epithelium, alveolar bone loss, and inflammatory cell infiltration in the connective tissue (Figure 1). The polymorphonuclear leukocytes were the main infiltrating cells. There were significant differences in the number of inflammatory cells, the distance between the cemento-enamel junction and the alveolar bone crest, and the ratio of TNF- $\alpha$-positive fibroblasts to total fibroblasts between the periodontal group and the control group (Table 2).

A number of accumulations of macrophage and lipid deposition in the descending aorta were observed in the periodontitis group but not in the control group (Figure 2). The mean (s.d.) percentage of aortic lumen occupied by the lesion was 3.0 (1.2) in the periodontitis group. $\mathrm{ROS}\left(\mathrm{H}_{2} \mathrm{O}_{2}\right)$
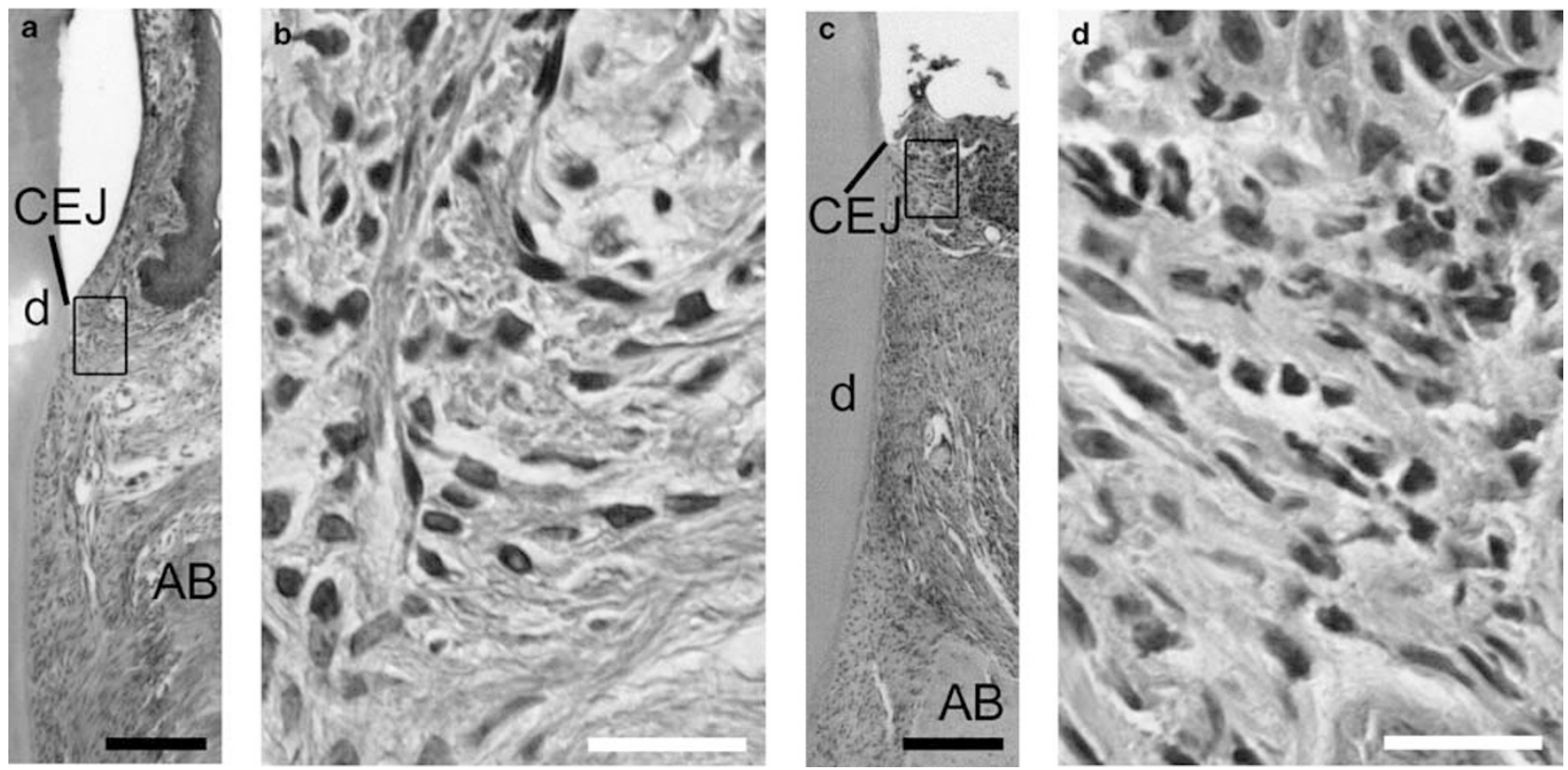

Figure 1 Pathological changes in rat periodontal tissue. The control group (a) with black boxes designating close-ups (b) showed few pathological changes. The periodontitis group (c) with black boxes designating close-ups (d) showed inflammatory cell infiltration in the connective tissue. Polymorphonuclear leukocytes were the main infiltrating cells. AB, alveolar bone; CEJ, cemento-enamel junction; $d$, dentin. Black scale bar $=200 \mu$ m. White scale bar $=40 \mu \mathrm{m}$. 
Table 2 Histomorphometrical analysis of rat periodontal tissue (Mean (s.d.))

\begin{tabular}{lcc}
\hline Parameters & Control $(n=6)$ & Periodontitis $(n=6)$ \\
\hline Linear distances between the cemento-enamel junction and the alveolar bone crest $(\mu \mathrm{m})$ & $591(232)$ & $3.0(0.8)$ \\
Polymorphonuclear leukocyte density (number/0.05 $\mathrm{mm} \times 0.1 \mathrm{~mm})$ & $0.12(0.07)$ & $6.8(1.1)^{*}$
\end{tabular}

${ }^{\star} P<0.05$, compared with the control group, according to the Mann-Whitney U-test.
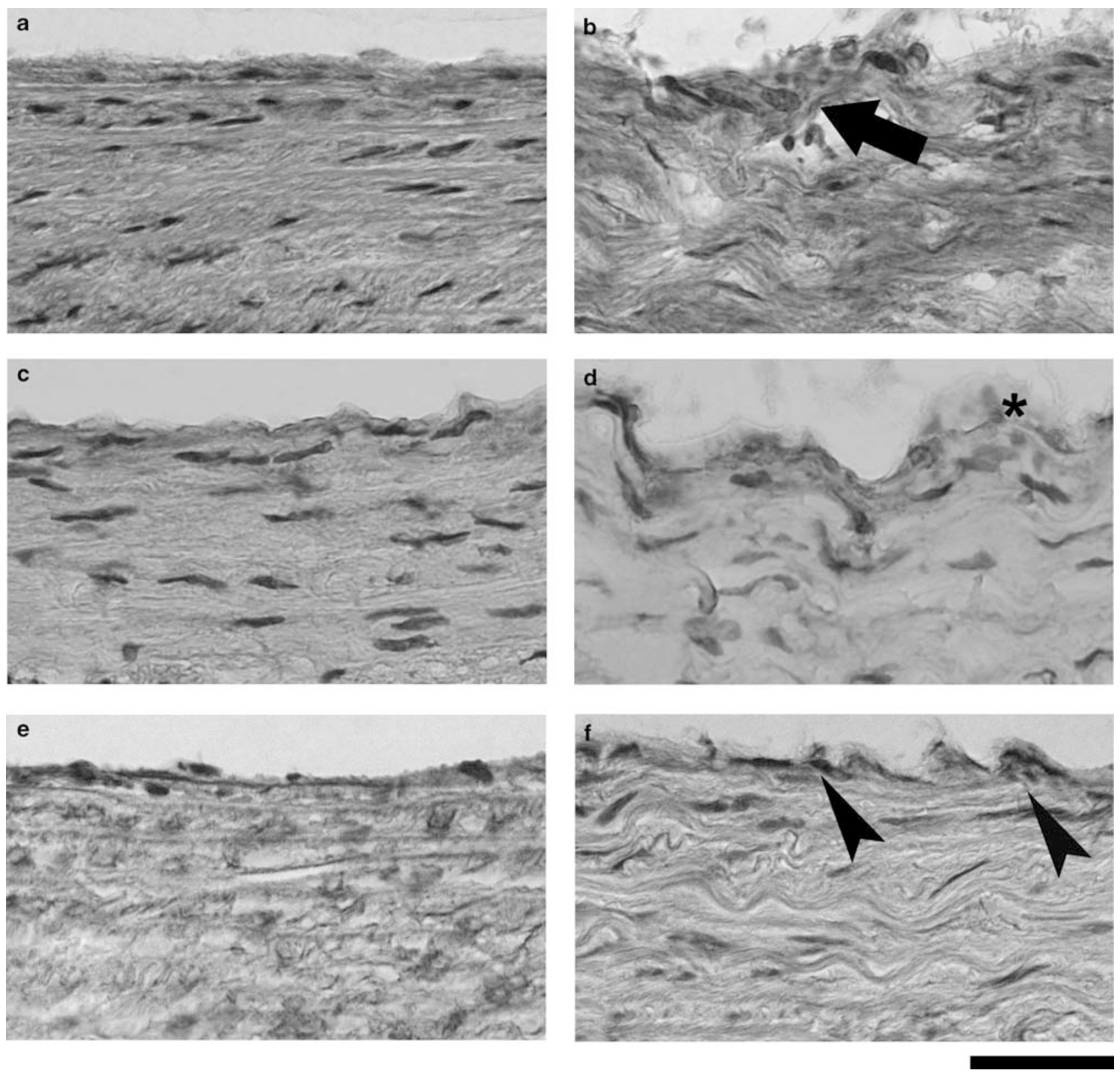

Figure 2 Representative results of initial stage of atherosclerosis, lipid deposition, and expression of reactive oxygen species $\left(\mathrm{H}_{2} \mathrm{O}_{2}\right)$ in the descending aorta. Macrophage collection (arrow) (b) and lipid deposition (asterisk) (d) was observed in the periodontitis group but not in the control group (a, c). $\mathrm{H}_{2} \mathrm{O}_{2}$ expression (arrowheads) in the periodontitis group (f) increased compared with that in the control group (e). Scale bar $=50 \mu \mathrm{m}$. 
expression in the periodontitis group increased compared with that in the control group (Figure 2).

In the aorta samples, four genes of all the 293 genes listed in the microarray showed more than twofold modification in response to periodontitis (Table 3). Level of endothelial NO synthase (eNOS) expression, which is related with insulin resistance did not change. Socs3 and Vegf mRNA expression was upregulated and Pi3kcb downregulated in both RT-PCR and microarray analysis (Table 4).

Weak Socs3 expression was detected in the descending aorta in the control group (Figure 3a). By contrast, in the periodontitis group, an increase in Socs3 expression was clearly evident (Figures $3 \mathrm{~b}$ and 4$)(P<0.05)$. The expression of Vcam1 and nitrotyrosine in the aortic lumen markedly increased in the periodontitis group compared with the control group (Figures $3 \mathrm{c}-\mathrm{f}$ and 4$) \quad(P<0.05)$, whereas those of Irs1, Irs2 and Pi3kp85 significantly decreased in the periodontitis group compared with the control group (Figures $3 \mathrm{~g}-\mathrm{l}$ and 4$)(P<0.05)$.

In the control group, insulin administration resulted in a noticeable increase in the p-Akt-positive area (Figure 5). Rats in the periodontitis group exhibited a reduced response because the phosphorylation of Akt after insulin stimulation was decreased $(P<0.05)$ compared with that in the control group.

Serum CRP level of the periodontitis group showed a $12 \%$ increase compared with that of the control group $(P<0.05)$ (Figure 6). Level of serum TNF- $\alpha$ in the periodontitis group was significantly higher than that in the control group
$(P<0.05)$ (Figure 7). There was no significant difference in serum insulin level between the periodontitis group (mean: 0.94 , s.d.: $0.15 \mu \mathrm{g} / \mathrm{l})$ and the control group $(0.86,0.15 \mu \mathrm{g} / \mathrm{l})$.

\section{DISCUSSION}

In our preliminary tests, we investigated the level of serum insulin and histological findings of periodontal tissues in Zucker fatty rats' lean littermates. The levels of serum insulin and inflammatory cell infiltration in Zucker fatty rats were significantly higher than those of lean rats (data not shown), which were similar to other studies. ${ }^{39,40}$ We have already reported that periodontitis induces the initial stage of atherosclerosis in normal rats. ${ }^{19}$ Therefore, we focused on the combined effects of periodontitis and obesity on aorta in this study.

As a result, the aorta samples in the periodontitis group of Zucker fatty rats showed accumulation of lipids, macrophage collection, increased Socs 3 and Vcam1 protein expression, decreased Irs1, Irs2, and Pi3kp85 protein expression, and changes of Socs3, Vcam1, Vegf, Pi3kcb, and Et-1 gene expression. These molecules are involved in aortic insulin resistance and endothelial dysfunction.

Expression of Socs 3 has been reported to increase in genetic rodent models of obesity and diabetes, in lesions of atherosclerosis, and in white adipose tissue of mice made obese by a high-fat diet. ${ }^{32,41,42}$ Socs 3 has been also proposed as a potential mediator of the insulin resistance induced by some hormones. ${ }^{43,44}$ SOCS proteins disrupt insulin signaling by binding to the insulin receptor and/or by targeting Irs 1

Table 3 Whole expression of genes is increased or decreased in aortic lesions of obese rats by periodontitis

\begin{tabular}{llll}
\hline Gene symbol & GenBank no. & Description & Fold up- or downregulation \\
\hline Socs3 & NM_053565 & Suppressor of cytokine signaling-3 & 3.6 \\
Hbegf & NM_012945 & Heparin-binding EGF-like growth factor & 2.8 \\
Ppargc1b & NM_176075 & Peroxisome proliferative activated receptor, $\gamma$, coactivator-1 $\beta$ & 0.5 \\
Fabp3 & NM_024162 & Fatty acid-binding protein-3 & 0.3 \\
\hline
\end{tabular}

Table 4 Fold changes in mRNA levels of aortic lesions in obese rats by periodontitis

\begin{tabular}{|c|c|c|c|c|}
\hline Gene symbol & GenBank no. & Description & Microarray & Real time RT-PCR \\
\hline Socs3 & NM_053565 & Suppressor of cytokine signaling-3 & 3.6 & 2.1 \\
\hline Vegf & NM_031836 & Vascular endothelial growth factor & 1.3 & 2.7 \\
\hline Et-1 & NM_012548 & Endothelin-1 & $N D^{a}$ & 2.2 \\
\hline
\end{tabular}

${ }^{\mathrm{a}}$ Vascular cell adhesion molecule-1 and Endothelin-1 were not listed in the microarray. 

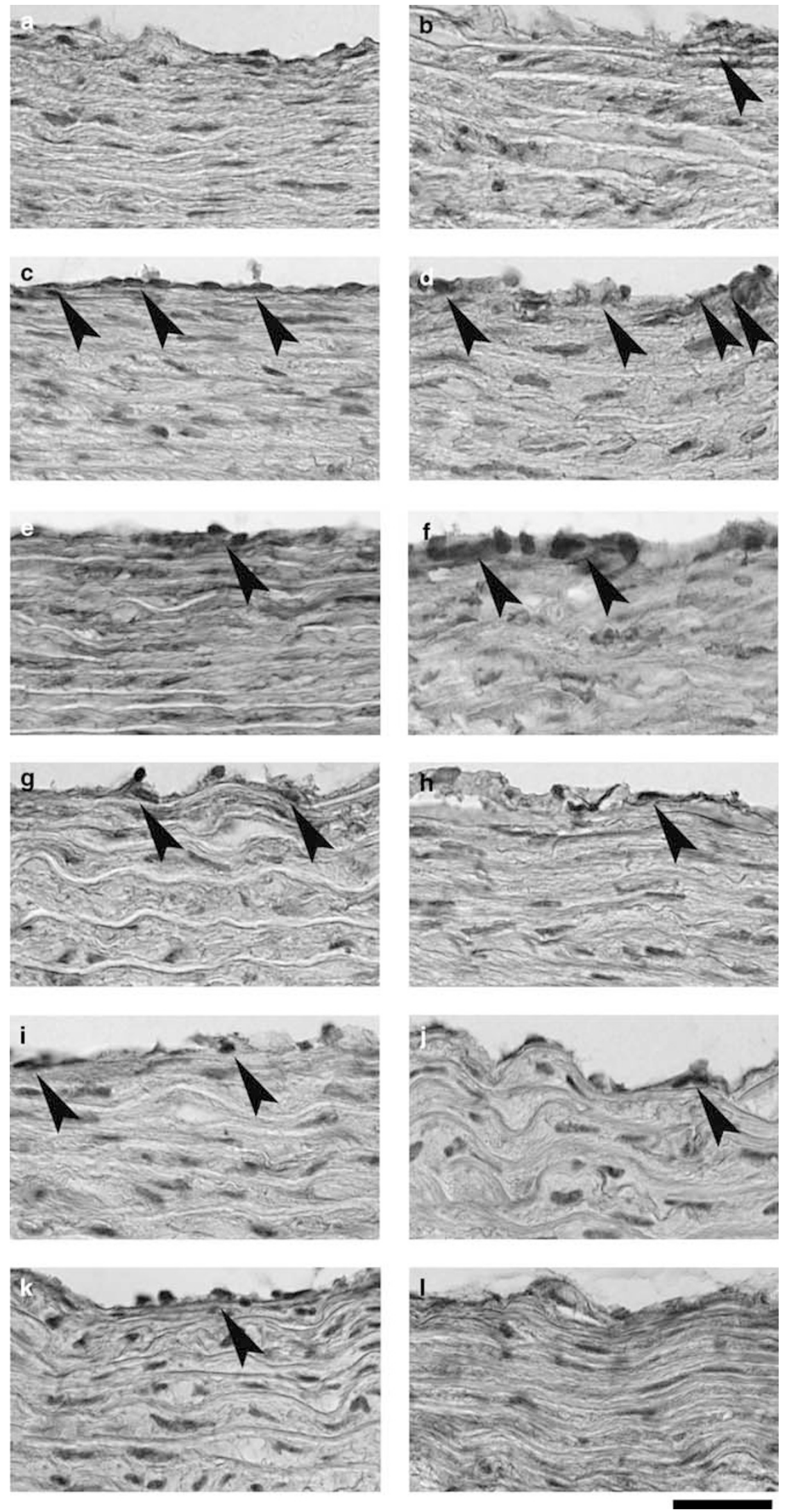

Figure 3 Cross-sections of descending aorta in the control and periodontitis groups. Positive areas of Socs3, Vcam1, and nitrotyrosine expression (black arrowheads) in the periodontitis group (b, d, $\mathbf{f}$ ) were more intense than in the control group (a, $\mathbf{c}, \mathbf{e})$. Irs 1 , Irs2, and Pi3kp85 expression areas in the control group ( $\mathbf{g}, \mathbf{i}, \mathbf{k})$ (black arrowheads) were more intense than in the periodontitis group $(\mathbf{h}, \mathbf{j}, \mathbf{I})$. Scale bar $=50 \mu \mathrm{m}$. 


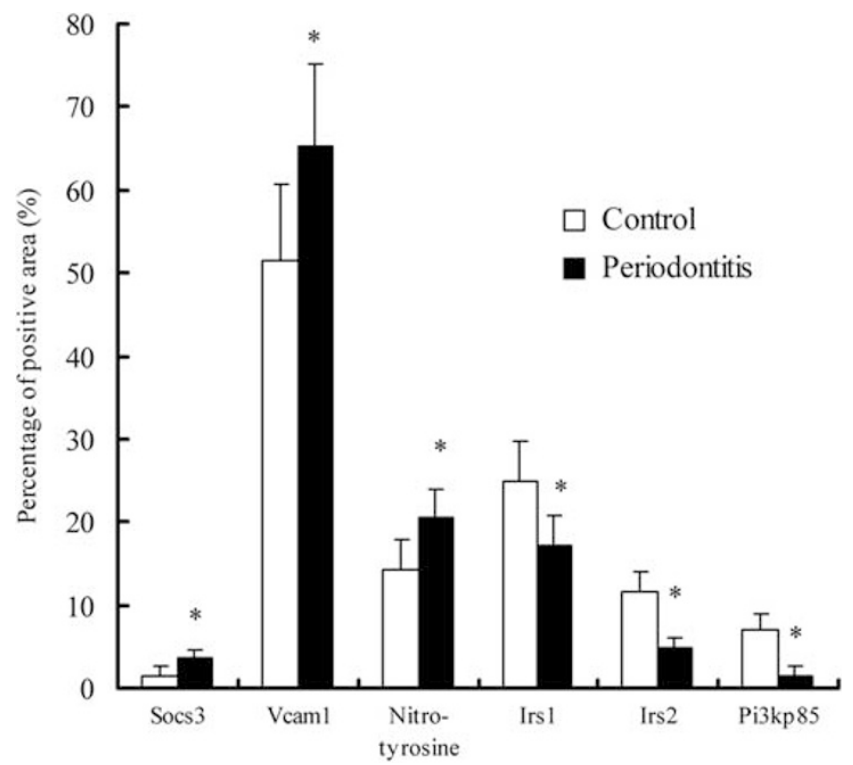

Figure 4 Percentage of Socs3-, Vcam1-, nitrotyrosine-, Irs1-, Irs2-, and Pi3kp85-positive areas of aortic lumen in rats. Means \pm s.d. of six rats. ${ }^{*} P<0.05$, compared with the control group, using the Mann-Whitney $U$-test.

and Irs2 for proteasomal degradation. In adipocytes, Socs3 reduced Irs1 phosphorylation and its subsequent association with Pi3kcb. ${ }^{45}$

Vegf, which are known to contribute to the proliferation of vascular smooth muscle cells, is enhanced in a model of metabolic insulin resistance with compensatory hyperinsulinemia. ${ }^{46}$ In addition, upregulation of endothelial cell adhesion molecules, Vcam1 and E-selectin, and increased rolling interactions of monocytes with endothelial cells are observed. ${ }^{46}$ Thus, compensatory hyperinsulinemia in the presence of metabolic insulin resistance with pathway-specific impairment of Pi3k in endothelium and vascular smooth muscle cells leads to enhanced mitogenic actions of insulin, which might contribute to the pathogenesis of atherosclerosis. ${ }^{47}$ These data support our hypothesis that periodontitis in obesity may affect the initiation of atherosclerosis by disturbing aortic insulin resistance.

Nitric oxide production and eNOS expression is associated with insulin resistance. Upregulation of eNOS was not observed in the periodontitis model. This result was supported by another model study, in which endotheliumspecific mutant insulin receptor overexpressing mice demonstrates similar levels of eNOS mRNA and protein expression to wild type. ${ }^{12}$ Insulin stimulates endothelial cell production of NO. ${ }^{10}$ Therefore, insulin resistance at the level of the endothelium might be associated with reduced insulin-stimulated NO. A mouse with endothelium-targeted deficiency of the insulin receptor is used to study the effect of insulin resistance specific to the endothelium on metabolic homeostasis. ${ }^{11}$ Recently, a study shows that selective endothelial insulin resistance is sufficient to induce a reduction in $\mathrm{NO}$ bioavailability and endothelial dysfunction that is secondary to increased generation of ROS. ${ }^{12}$ Peroxynitrite produced by the reaction between $\mathrm{NO}$ and ROS may itself exert toxic effects through protein nitration and contribute to a reduction in the bioavailability of $\mathrm{NO}^{21}$ Increased level of ROS and nitrotyrosine were observed in the aorta of the periodontitis group compared with those of the control group. These results may be related with aortic insulin resistance. $^{12}$

Rats with periodontitis showed increased nitrotyrosine and Et-1 expression. Endothelial dysfunction refers to an imbalance in the release of vasodilating factors such as NO and vasoconstricting factors such as Et-1. ${ }^{9}$ Under the condition of insulin resistance, downregulation of $\mathrm{NO}$ production, which is related to the increased level of nitrotyrosine, and upregulation of Et-1 production are detected. An imbalance between these factors may be responsible for initiation of arteriosclerosis in this study.

In the aorta of the periodontitis group, the phosphorylation of Akt after insulin stimulation was decreased compared with that in the control group. After insulin administration, Zucker fatty rats show a decreased phosphorylation of Akt in liver compared with the lean rats, which reflects local insulin resistance. ${ }^{48}$ These suggest that periodontal inflammation in the obese rats leads to aortic insulin resistance.

Our study used a ligature-induced periodontitis model, because such a periodontal lesion mimics several features of human periodontitis, including the inflammatory cellular infiltrate and alveolar bone resorption. ${ }^{19,22,49}$ We confirmed these findings in the periodontitis group. The number of polymorphonuclear leukocytes in our study (6.8 (1.1) numbers $/ 0.05 \mathrm{~mm} \times 0.1 \mathrm{~mm})$ was similar to that $(7.4(1.7)$ numbers $/ 0.05 \mathrm{~mm} \times 0.1 \mathrm{~mm})$ of the previous studies. ${ }^{21}$ However, the distance between the cemento-enamel junction and the alveolar bone crest in this study (869 (128) $\mu \mathrm{m})$ was greater than that $(579(63) \mu \mathrm{m})$ in the previous data. ${ }^{50} \mathrm{Be}-$ cause we used obese model rats and the previous studies normal rats, the difference of data of alveolar bone loss may depend on the rat model used in each study.

Tumor necrosis factor- $\alpha$ expression in the periodontal tissue of the periodontitis group was higher than that of the control group. The previous study shows increased TNF- $\alpha$ expression in the periodontal tissue using the same ligature model with increased level of serum TNF- $\alpha{ }^{51}$ TNF- $\alpha$ is induced in inflammatory tissues and CRP is produced in the liver stimulated by cytokines secreted in the inflammatory tissues. Enhanced TNF- $\alpha$ expression in the periodontal tissue may contribute to increased levels of serum TNF- $\alpha$ and CRP.

Serum levels of CRP and TNF- $\alpha$ in the periodontitis group significantly increased compared with the control group in this study. High-sensitivity CRP has received much attention and several studies support a strong link between baseline elevations of high-sensitivity CRP and future risk of coronary events. ${ }^{52,53}$ Several reports have demonstrated a statistically significant increase in serum CRP levels in periodontitis 

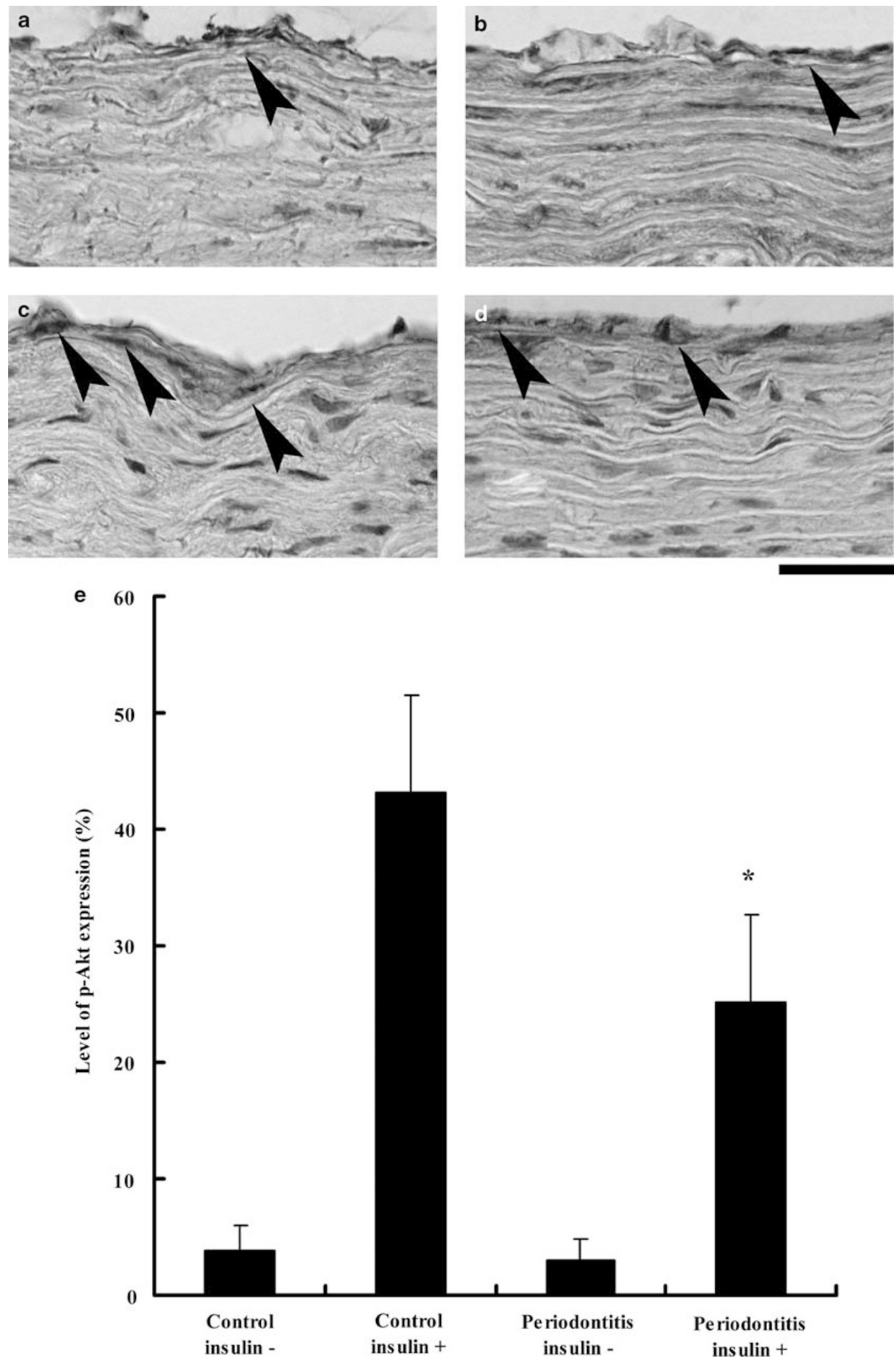

Figure 5 Percentage of p-Akt-positive areas of aortic lumen in rats. There was no markedly difference in p-Akt expression (black arrowheads) between the control group (insulin -) (a) and periodontitis group (insulin -) (b). After insulin administration, the positive areas of p-Akt expression in the control group (c) were more intense than in the periodontitis group (d). Panel e shows the percentage of p-Akt-positive areas of aortic lumen in rats (means \pm s.d. of six rats). Scale bar $=50 \mu \mathrm{m}$. ${ }^{\star P}<0.05$, compared with the control group (insulin + ), using the Mann-Whitney U-test. 


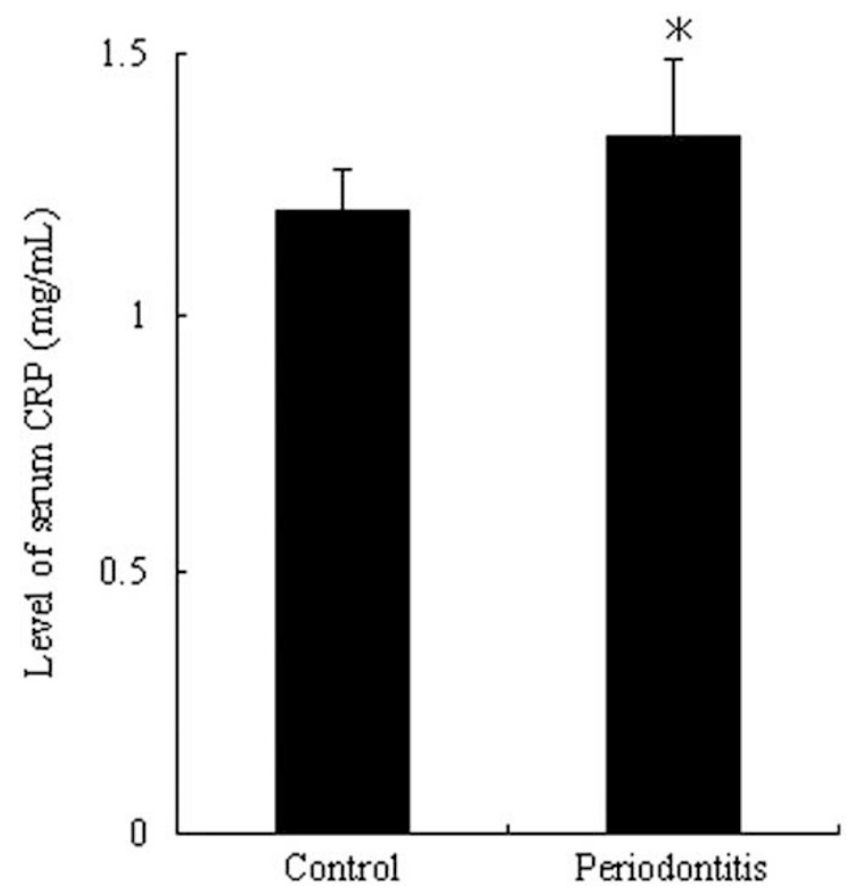

Figure 6 Serum CRP level in rats. Means \pm s.d. of six rats. ${ }^{\star} P<0.05$, compared with the control group, using the Mann-Whitney $U$-test.

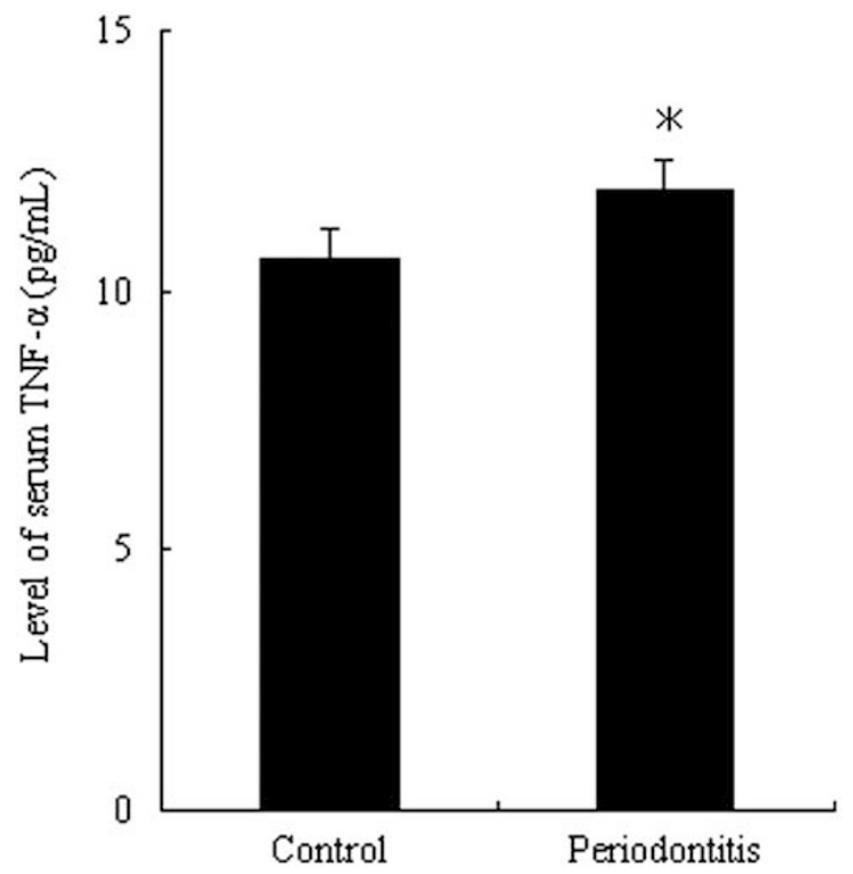

Figure 7 Serum TNF- $\alpha$ level in rats. Means \pm s.d. of six rats. ${ }^{\star} P<0.05$, compared with the control group, using the Mann-Whitney $U$-test.

patients compared with non-diseased control subjects. ${ }^{54,55}$ CRP is also correlated to the presence of both insulin resistance and endothelial dysfunction. ${ }^{56}$ Other proinflammatory cytokines, such as TNF- $\alpha$, are also important in the inflammatory process. Elevated levels of TNF- $\alpha$ may be associated with increased risk for future development of atherosclerosis in periodontitis patients with type 2 diabetes mellitus. ${ }^{57,58} \mathrm{TNF}-\alpha$ is closely associated with not only insulin resistance but also with vascular atherogenic changes. ${ }^{59,60}$

SOSC genes are positively induced by cytokines. ${ }^{61,62}$ Several reports have shown that Socs3 by inflammatory cytokines desensitizes insulin signaling by targeting Irs1/Irs2 for degradation, leading to the development of obesity, hepatic steatosis, or metabolic syndrome. ${ }^{45,63,64}$ Increased levels of CRP and TNF- $\alpha$ in the periodontitis group in the obesity model may contribute to aortic insulin resistance and initial stage of atherosclerosis, through upregulation of Socs3 and downregulation of Irs1 and Irs2.

There was no difference in the serum level of insulin between the periodontitis group and the control group in normal-diet fed Zucker fatty rats. The result was consistent with another study using the same model. ${ }^{65}$ In the previous study, ${ }^{65}$ periodontitis accelerates the onset of severe systemic insulin resistance and impaired glucose homeostasis in highfat fed Zucker fatty rats, but not in low-fat fed Zucker rats. Systemic insulin resistance might not be observed in our study with normal-fat fed rats. However, we did not evaluate the systemic insulin resistance such as homeostasis model assessment and glucose tolerance test. In future studies, systemic insulin resistance and glucose tolerance test should be included using high-fat fed Zucker fatty rats, as the response of local and systemic insulin resistance differs. ${ }^{66}$

Aorta samples of the control Zucker fatty rats showed no initial lesion of atherosclerosis in this study. Zucker fatty rats do not develop atherosclerosis spontaneously. ${ }^{67,68}$ However, Zucker fatty rats fed on a human-type atherogenic diet shows atherosclerotic lesions. Atherogenesis starts with lipid insudation into the vessel wall, where it is trapped in the intima. The endothelium of the site where the lipid is trapped becomes dysfunctional, resulting in monocytes rolling and then adhering to the endothelium, before migrating into the intima, resulting in macrophage collection. ${ }^{69}$ The lipid deposition and macrophage collection corresponding to an initial stage of atherosclerosis were observed in the periodontitis Zucker fatty rats. Therefore, Zucker fatty rats are suitable for various kinds of experimental studies on atherosclerosis. ${ }^{70}$

Our study has some limitations. First, we used a ligatureinduced periodontitis model because such a periodontal lesion mimics several features of human periodontitis. However, ligature-induced periodontal inflammation is an acute model of periodontitis and is not equivalent to the chronic disease in humans. In fact, polymorphonuclear leukocytes were the main infiltrating cells and a few plasma cells were observed in periodontal lesions in the periodontitis group in this study. Second, sham control groups that received saline without insulin were not used.

In conclusion, periodontitis in obesity induced the initial stage of atherosclerosis and disturbed aortic insulin signaling 
through increased levels of Socs3, Et-1, ROS and nitrotyrosine, and decreased levels of Pi3kcb, Pi3kp85, Irs1, Irs2.

\section{ACKNOWLEDGEMENT}

We thank Professor Masaharu Takigawa, Department of Biochemistry and Molecular Dentistry, Okayama University Graduate School of Medicine, Dentistry and Pharmaceutical Sciences, Japan, for his valuable comments regarding real-time PCR. This study was supported by Grants-in-aid for Scientific Research $(20791642,21792148,21792150)$ from the Ministry of Education, Culture, Sports, Science and Technology, Tokyo, Japan.

\section{DISCLOSURE/CONFLICT OF INTEREST}

The authors declare no conflict of interest.

1. Reilly MP, Rader DJ. The metabolic syndrome: more than the sum of its parts? Circulation 2003;108:1546-1551.

2. Hayden JM, Reaven PD. Cardiovascular disease in diabetes mellitus type 2: a potential role for novel cardiovascular risk factors. Curr Opin Lipidol 2000;11:519-528.

3. Sowers JR. Obesity as a cardiovascular risk factor. Am J Med 2003:115:37S-41S.

4. Hanley AJ, Williams K, Stern MP, et al. Homeostasis model assessment of insulin resistance in relation to the incidence of cardiovascular disease: the San Antonio Heart Study. Diabetes Care 2002;25: 1177-1184.

5. Haffner SM, D'Agostino Jr R, Mykkänen L, et al. Insulin sensitivity in subjects with type 2 diabetes. Relationship to cardiovascular risk factors: the Insulin Resistance Atherosclerosis Study. Diabetes Care 1999;22:562-568.

6. Sinaiko AR, Steinberger J, Moran A, et al. Relation of body mass index and insulin resistance to cardiovascular risk factors, inflammatory factors, and oxidative stress during adolescence. Circulation 2005;111:1985-1991.

7. Prior JO, Quinones MJ, Hernandez-Pampaloni M, et al. Coronary circulatory dysfunction in insulin resistance, impaired glucose tolerance, and type 2 diabetes mellitus. Circulation 2005;111: 2291-2298.

8. Tousoulis D, Tsarpalis $K$, Cokkinos D, et al. Effects of insulin resistance on endothelial function: possible mechanisms and clinical implications. Diabetes Obes Metab 2008;10:834-842.

9. Kim JA, Montagnani M, Koh KK, et al. Reciprocal relationships between insulin resistance and endothelial dysfunction: molecular and pathophysiological mechanisms. Circulation 2006;113:1888-1904.

10. Zeng G, Nystrom FH, Ravichandran LV, et al. Roles for insulin receptor, $\mathrm{PI}$-kinase, and Akt in insulin-signaling pathways related to production of nitric oxide in human vascular endothelial cells. Circulation 2000;101:1539-1545.

11. Vicent $D$, llany J, Kondo $T$, et al. The role of endothelial insulin signaling in the regulation of vascular tone and insulin resistance. J Clin Invest 2003;111:1373-1380.

12. Duncan ER, Crossey PA, Walker $S$, et al. Effect of endothelium-specific insulin resistance on endothelial function in vivo. Diabetes 2008;57:3307-3314.

13. Page RC, Engel LD, Narayanan AS, et al. Chronic inflammatory gingival and periodontal disease. JAMA 1978;11:545-550.

14. Pihlstrom BL, Michalowicz BS, Johnson NW. Periodontal diseases. Lancet 2005;366:1809-1820.

15. Cueto A, Mesa F, Bravo M, et al. Periodontitis as risk factor for acute myocardial infarction. A case control study of Spanish adults. J Periodontal Res 2005;40:36-42.

16. Janket SJ, Baird AE, Chuang SK, et al. Meta-analysis of periodontal disease and risk of coronary heart disease and stroke. Oral Surg Oral Med Oral Pathol Oral Radiol Endod 2003;95:559-569.

17. Söder PO, Söder B, Nowak J, et al. Early carotid atherosclerosis in subjects with periodontal diseases. Stroke 2005;36:1195-1200.

18. Spahr A, Klein E, Khuseyinova N, et al. Periodontal infections and coronary heart disease: role of periodontal bacteria and importance of total pathogen burden in the Coronary Event and Periodontal Disease (CORODONT) study. Arch Intern Med 2006;166:554-559.
19. Ekuni D, Tomofuji T, Sanbe T, et al. Periodontitis-induced lipid peroxidation in rat descending aorta is involved in the initiation of atherosclerosis. J Periodontal Res 2009;44:434-442.

20. Janssen U, Phillips AO, Floege J. Rodent models of nephropathy associated with type II diabetes. J Nephrol 1999;12:159-172.

21. Kearney MT, Duncan ER, Kahn M, et al. Insulin resistance and endothelial cell dysfunction: studies in mammalian models. Exp Physiol 2008;93:158-163.

22. Irie K, Tomofuji T, Tamaki N, et al. Effects of ethanol consumption on periodontal inflammation in rats. J Dent Res 2008;87:456-460.

23. Torsoni MA, Carvalheira JB, Pereira-Da-Silva $M$, et al. Molecular and functional resistance to insulin in hypothalamus of rats exposed to cold. Am J Physiol Endocrinol Metab 2003;285:E216-E223.

24. Ekuni D, Yamamoto T, Yamanaka R, et al. Proteases augment the effects of lipopolysaccharide in rat gingiva. J Periodontal Res 2003;38:591-596.

25. Kawata K, Eguchi T, Kubota S, et al. Possible role of LRP1, a CCN2 receptor, in chondrocytes. Biochem Biophys Res Commun 2006;345:552-559.

26. Peinnequin $A$, Mouret $C$, Birot $O$, et al. Rat pro-inflammatory cytokine and cytokine related mRNA quantification by real-time polymerase chain reaction using SYBR green. BMC Immunol 2004;5:3.

27. Simler N, Grosfeld A, Peinnequin A, et al. Leptin receptor-deficient obese Zucker rats reduce their food intake in response to hypobaric hypoxia. Am J Physiol Endocrinol Metab 2006;290:E591-E597.

28. Uruno A, Sugawara A, Kanatsuka $H$, et al. Upregulation of nitric oxide production in vascular endothelial cells by all-trans retinoic acid through the phosphoinositide 3-kinase/Akt pathway. Circulation 2005;112:727-736.

29. Berti R, Williams AJ, Moffett JR, et al. Quantitative real-time RT-PCR analysis of inflammatory gene expression associated with ischemiareperfusion brain injury. J Cereb Blood Flow Metab 2002;22: 1068-1079.

30. Fukuda G, Khan ZA, Barbin YP, et al. Endothelin-mediated remodeling in aortas of diabetic rats. Diabetes Metab Res Rev 2005;21: 367-375.

31. Ekuni D, Tomofuji T, Sanbe T, et al. Vitamin C intake attenuates the degree of experimental atherosclerosis induced by periodontitis in the rat by decreasing oxidative stress. Arch Oral Biol 2009;54:495-502.

32. Tang J, Kozaki K, Farr AG, et al. The absence of platelet-derived growth factor-B in circulating cells promotes immune and inflammatory responses in atherosclerosis-prone ApoE-/- mice. Am J Pathol 2005;167:901-912.

33. Escribese MM, Conde E, Martín A, et al. Therapeutic effect of all-transretinoic acid (at-RA) on an autoimmune nephritis experimental model: role of the VLA-4 integrin. BMC Nephrol 2007;8:3.

34. Kokk K, Veräjänkorva $E, W u X K$, et al. Expression of insulin signaling transmitters and glucose transporters at the protein level in the rat testis. Ann N Y Acad Sci 2007;1095:262-273.

35. Cai F, Helke CJ. Abnormal PI3 kinase/Akt signal pathway in vagal afferent neurons and vagus nerve of streptozotocin-diabetic rats. Brain Res Mol Brain Res 2003;110:234-244.

36. Wang R, Zhang QG, Han D, et al. Inhibition of MLK3-MKK4/7-JNK1/2 pathway by Akt1 in exogenous estrogen-induced neuroprotection against transient global cerebral ischemia by a non-genomic mechanism in male rats. J Neurochem 2006;99:1543-1554.

37. Tomofuji T, Ekuni D, Yamanaka R, et al. Chronic administration of lipopolysaccharide and proteases induces periodontal inflammation and hepatic steatosis in rats. J Periodontol 2007;78:1999-2006.

38. Ackermans MT, Klieverik LP, Endert E, et al. Plasma insulin concentrations during a hyperinsulinaemic clamp: what do we measure? Ann Clin Biochem 2008;45:429-430.

39. Chiba T, Komatsu T, Nakayama M, et al. Similar metabolic responses to calorie restriction in lean and obese Zucker rats. Mol Cell Endocrinol 2009;309:17-25.

40. Liu R, Bal HS, Desta $T$, et al. Diabetes enhances periodontal bone loss through enhanced resorption and diminished bone formation. J Dent Res 2006;85:510-514.

41. Bjørbaek C, Elmquist JK, Frantz JD, et al. Identification of SOCS-3 as a potential mediator of central leptin resistance. Mol Cell 1998;1:619-625.

42. Wang ZW, Pan WT, Lee Y, et al. The role of leptin resistance in the lipid abnormalities of aging. FASEB J 2001;15:108-114. 
43. Shi $\mathrm{H}$, Tzameli I, Bjørbaek $\mathrm{C}$, et al. Suppressor of cytokine signaling 3 is a physiological regulator of adipocyte insulin signaling. J Biol Chem 2004:279:34733-34740.

44. Steppan CM, Wang J, Whiteman EL, et al. Activation of SOCS-3 by resistin. Mol Cell Biol 2005;25:1569-1575.

45. Emanuelli $B$, Peraldi $P$, Filloux $C$, et al. SOCS-3 inhibits insulin signaling and is up-regulated in response to tumor necrosis factor-alpha in the adipose tissue of obese mice. J Biol Chem 2001;276:47944-47949.

46. Montagnani M, Golovchenko I, Kim I, et al. Inhibition of phosphatidylinositol 3-kinase enhances mitogenic actions of insulin in endothelial cells. J Biol Chem 2002;277:1794-1799.

47. Kim JA, Montagnani M, Koh KK, et al. Reciprocal relationships between insulin resistance and endothelial dysfunction: molecular and pathophysiological mechanisms. Circulation 2006;113:1888-1904.

48. Muñoz MC, Argentino DP, Dominici FP, et al. Irbesartan restores the in-vivo insulin signaling pathway leading to Akt activation in obese Zucker rats. J Hypertens 2006;24:1607-1617.

49. Di Paola R, Marzocco S, Mazzon E, et al. Effect of aminoguanidine in ligature-induced periodontitis in rats. J Dent Res 2004;83:343-348.

50. Tomofuji T, Ekuni D, Sanbe T, et al. Effects of vitamin C intake on gingival oxidative stress in rat periodontitis. Free Radic Biol Med 2009;46:163-168.

51. Tomofuji T, Ekuni D, Irie K, et al. Preventive effects of a cocoa-enriched diet on gingival oxidative stress in experimental periodontitis. J Periodontol 2009;80:1799-1808.

52. Ridker PM, Cushman M, Stampfer MJ et al. Inflammation, aspirin, and the risk of cardiovascular disease in apparently healthy men N Engl J Med 1997;336:973-979.

53. Ridker PM, Cushman M, Stampfer MJ, et al. Plasma concentration of C-reactive protein and risk of developing peripheral vascular disease. Circulation 1998:97:425-428.

54. Noack B, Genco RJ, Trevisan M, et al. Periodontal infections contribute to elevated systemic C-reactive protein level. J Periodontol 2001;72:1221-1227.

55. Craig RG, Yip JK, So MK, et al. Relationship of destructive periodontal disease to the acute-phase response. J Periodontol 2003;74: 1007-1016.

56. Yudkin JS, Stehouwer CD, Emeis JJ, et al. C-reactive protein in healthy subjects: associations with obesity, insulin resistance, and endothelial dysfunction: a potential role for cytokines originating from adipose tissue? Arterioscler Thromb Vasc Biol 1999;19:972-978.
57. Iwamoto $Y$, Nishimura $F$, Nakagawa $M$, et al. The effect of antimicrobial periodontal treatment on circulating tumor necrosis factor-alpha and glycated hemoglobin level in patients with type 2 diabetes. J Periodontol 2001;72:774-778.

58. Iwamoto $Y$, Nishimura F, Soga $Y$, et al. Antimicrobial periodontal treatment decreases serum C-reactive protein, tumor necrosis factoralpha, but not adiponectin levels in patients with chronic periodontitis. J Periodontol 2003;74:1231-1236.

59. Nishimura F, Murayama Y. Periodontal inflammation and insulin resistance-lessons from obesity. J Dent Res 2001;80:1690-1694.

60. Barath P, Fishbein MC, Cao J, et al. Detection and localization of tumor necrosis factor in human atheroma. Am J Cardiol 1990;65:297-302.

61. Starr R, Willson TA, Viney EM, et al. A family of cytokine-inducible inhibitors of signalling. Nature 1997;387:917-921.

62. Emanuelli B, Peraldi P, Filloux C, et al. SOCS-3 is an insulin-induced negative regulator of insulin signaling. J Biol Chem 2000;275: 15985-15991.

63. Ueki K, Kondo T, Kahn CR. Suppressor of cytokine signaling 1 (SOCS-1) and SOCS-3 cause insulin resistance through inhibition of tyrosine phosphorylation of insulin receptor substrate proteins by discrete mechanisms. Mol Cell Biol 2004:24:5434-5446.

64. Rui L, Yuan M, Frantz D, et al. SOCS-1 and SOCS-3 block insulin signaling by ubiquitin-mediated degradation of IRS1 and IRS2. J Biol Chem 2002;277:42394-42398.

65. Watanabe K, Petro BJ, Shlimon AE, et al. Effect of periodontitis on insulin resistance and the onset of type 2 diabetes mellitus in Zucker diabetic fatty rats. J Periodontol 2008;79:1208-1216.

66. Shi H, Cave B, Inouye K, et al. Overexpression of suppressor of cytokine signaling 3 in adipose tissue causes local but not systemic insulin resistance. Diabetes 2006;55:699-707.

67. Kasiske BL, O'Donnell MP, Keane WF. The Zucker rat model of obesity, insulin resistance, hyperlipidemia, and renal injury. Hypertension 1992;19:1110-I115.

68. Shimamura T, Amorosa LF, Wilson AC, et al. Atherosclerosis and glomerulosclerosis in WHHL rabbits and obese Zucker rats. Jpn J Exp Med 1990;60:105-109.

69. Kavurma MM, Bennett MR. Expression, regulation and function of trail in atherosclerosis. Biochem Pharmacol 2008:75:1441-1450.

70. Vaskonen T, Mervaala E, Krogerus L, et al. Supplementation of plant sterols and minerals benefits obese Zucker rats fed an atherogenic diet. J Nutr 2002;132:231-237. 